\title{
A Novel Pixel Vertex Detector for the Belle II Experiment at SuperKEKB
}

\section{Christian Kiesling ${ }^{* \dagger}$}

Max-Planck-Institute for Physics, Munich, Germany

E-mail: cmk@mpp.mpg.de

\begin{abstract}
At the KEK laboratory in Tsukuba, Japan, the upgrade of KEKB to "SuperKEKB" has started. Due to the much higher backgrounds expected at SuperKEKB, a massive upgrade of the Belle detector ("Belle II") is in progress as well. For the innermost layers, close to the beam pipe, strip sensors will not work anymore due to the large occupancies, and a pixel detector becomes mandatory for Belle II. We report here on the design and construction of a novel silicon pixel detector for Belle II, based on the DEPFET ("depleted $p$-channel $f$ ield $e$ ffect $t$ ransistor") technology. This technology, invented at the Max-Planck-Institute for Physics and produced in the Semiconductor Labororatory of the Max-Planck-Society, allows for very thin (down to $50 \mu \mathrm{m}$ ) sensors, which can be produced as large (10's of square centimeters) selfsupporting structures. Due to its internal amplification, the DEPFET pixel has low noise, a large S/N ratio and, finally, a low power consumption. A special set of ASICs at the ends of the sensors controls the digitzation of the FET current for readout and provides common mode and pedestal subtraction as well as zero-suppression. While the ASICs need active cooling by a two-phase $\mathrm{CO}_{2}$ system, the sensor area inside the physical acceptance region is only cooled by an airflow. First thin DEPFET matrices have been produced and tested successfully, using prototype ASICs mounted on externals PCBs and connected to the sensor via wire bonds. The performance of the matrices is as expected from simulations.
\end{abstract}

The 2011 Europhysics Conference on High Energy Physics-HEP 2011,

July 21-27, 2011

Grenoble, Rhône-Alpes France

${ }^{*}$ Speaker.

${ }^{\dagger}$ for the DEPFET Collaboration 


\section{Introduction}

Although the Standard Model (SM) has been extremely successful in describing virtually all experimental data so far, most importantly the $\mathrm{CP}$ violation phenomena of the $K$ - and the $B$-meson systems (for a comprehensive overview see, e.g. [1]), there are a number of compelling arguments why the SM cannot be regarded as the ultimate theory. In fact, there is clear evidence for physics beyond the SM, as suggested by the non-vanishing mass of the neutrinos, the apparent necessity for dark matter, and, most importantly perhaps, the huge matter-antimatter asymmetry observed in the universe, Most of the effects have to do with $\mathrm{CP}$ violation by a yet undiscovered source. The "New Physics" (NP) generating these sources is expected to appear at large, so far unreachable (multi-TeV) energy scales. While the discovery and exploration of NP thresholds (the "high energy frontier") is the central motivation for the LHC program, flavor physics with large data samples (the "intensity frontier") is expected to play a key role in unraveling possible NP at these scales and to solve the puzzle of $\mathrm{CP}$ violation.

\section{Physics Motivation for the SuperKEKB Project}

Some tantalizing hints for possible New Physics in the decays of $B$ mesons (see, e.g., [2]) have been observed, however still with insufficient significance. New insight can only come from a next generation of experiments, aiming at much larger events samples than have been obtained so far (typically half a billion $B$ decays at the present B-Factories).

In a "Super Flavor Factory", (SFF) [3, 4], instantaneous luminosities will be obtained which exceed the presently achievable world record value of $2 \times 10^{34}$ (obtained with KEKB) by almost two orders of magnitude. A recent review of the physics potential of a future high luminosity $B$ factory can be found, e.g., in [5]. One should note here that the discovery potential of a future SFF is indeed extraordinary and might probe new mass scales even beyond those reachable at the LHC. Concerning the SuperKEKB machine [3], the Japanese government has given full approval for the construction, which will be completed by late 2014 .

\section{Detector Upgrade: BELLE II and the PXD}

In the last year, the Technical Design Report [6] for the Belle upgrade ("Belle II") was published. The main (re)design goals are to cope with the much higher physics rates and the much larger backgrounds to be expected, as well as improving the overall physics performance.

A comparison of Belle and its upgraded version ("Belle II") is shown in a side view in fig. 1. The main changes with respect to Belle are the following: Particle identification (PID) will be improved by two types of detectors: One is a time-of-propagation (TOP) counter for the barrel region (Barrel PID), and the other a proximity-focusing Cerenkov ring imaging counter with aerogel radiators (ARICH) for the endcap region (Endcap PID). The magnet and the barrel part of the electromagnetic calorimeter (ECL) will not be changed, but equipped with new electronics with shorter shaping time to cope with the pileup from background. In the endcap parts of the ECL the present $\mathrm{CsI}(\mathrm{Tl})$ crystals will eventually be replaced by pure CsI that provide faster signals, and the forward part and the innermost layers of the barrel part of the $\operatorname{KLM~}\left(K_{L}\right.$ and muon detector) in the iron flux 


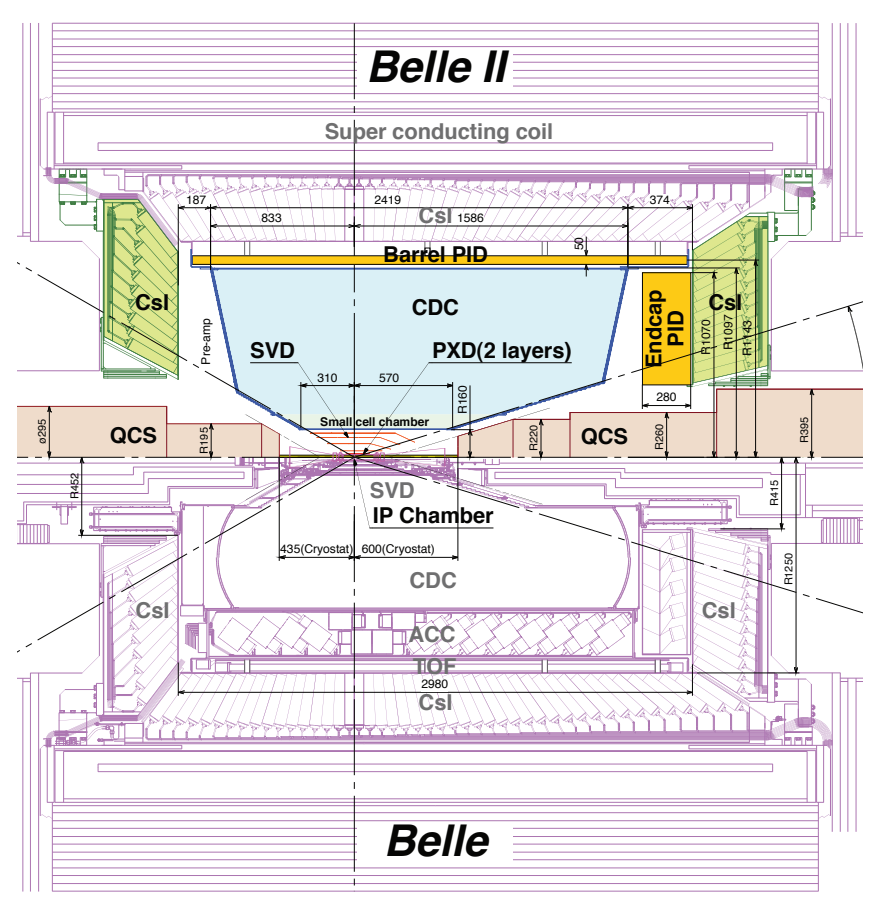

Figure 1: Upgrade scenario from the Belle detector (lower half) to the Belle II detector (upper half).

return yoke, closing the magnetic flux from the superconducting solenoid, will be instrumented by scintillator strips with SiPM readout, replacing the present RPCs.

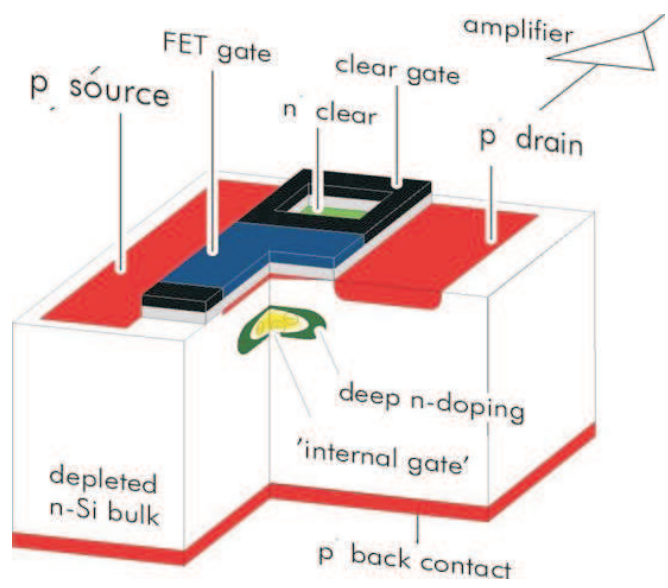

Figure 2: Principle of operation of the DEPFET pixel cell.

Furthermore, the entire tracking system will be replaced with a new small-cell drift chamber (CDC), surrounding a 4-layer Si-strip detector with double-sided readout (SVD). Closest to the beampipe, a two-layer Si-pixel detector (PXD) will be inserted. The PXD has to be thin (to minimize multiple scattering), radiation-hard, and must be read out with high speed. Presently, only the DEPFET pixel sensor ( $D E$ leted $P$-channel $F$ ield $E$ ffect $T$ ransistor) [7] fulfills all these criteria. Its principle of operation is shown in fig. 2: A $p$-channel MOSFET is integrated onto a silicon substrate forming the detector, which is fully depleted by a sufficiently high voltage. By means of an additional $n$-implant at a depth of about $1 \mu \mathrm{m}$ underneath the transistor channel a potential minimum is created ("internal gate"), The electrons liberated by particles traversing the depleted bulk are collected in the internal gate, while the holes drift to the negative back contact. 
When the transistor is switched on, the electrons in the internal gate modulate the channel current, After readout the signal charge in the internal gate is removed by a "clear" pulse. The transistor can then be switched on again, this time reading a current without charge in the internal gate. The signal is provided by the difference of the two currents. An important feature of the DEPFET is the very small capacitance of the internal gate so that the pixel can be operated with very low noise even at room temperature. The figure-of-merit of the DEPFET is its internal amplification, which is defined as the current response to an electron collected in the internal gate. Prototypes with an optimized channel geometry reach vales of about $500 \mathrm{pA} / \mathrm{electron}$ and beyond.

Arrays of DEPFET pixels can be fabricated as large modules, e.g. with 250 times 768 pixels, as foreseen for the Belle II PXD (see fig. 3 for an overview of the PXD layout). The pixel sizes are typically 50 times $70 \mu \mathrm{m}^{2}$. The arrays are read out row by row ("rolling shutter" mode), where the rows are consecutively switched on by an external gate ("Switcher"). The drain currents are digitized in a total of four 64-channel ASICs, the so-called "drain current digitizers" (DCD), which are located at the end of the sensor, outside of the acceptance region. The entire switchon, digitize and clear cycle for a row is less than $100 \mathrm{~ns}$, and an the array of 768 rows times 250 columns is read out within $20 \mu \mathrm{s}$, switching on 4 consecutive rows at a time, each of the columns being connected individually to its own DCD channel, The DCD is followed by the so-called "data handling processor" (DHP), which performs the common-mode and pedestal subtraction as well as the zero-suppression, using adjustable calibration constants, stored locally on the chip. The DHP also buffers the data and transmits to the data acquisition only those frames which are associated with a triggered event. In addition to the data processing task the DHP provides the timing signals for the synchronization of the Switchers and the DCDs.

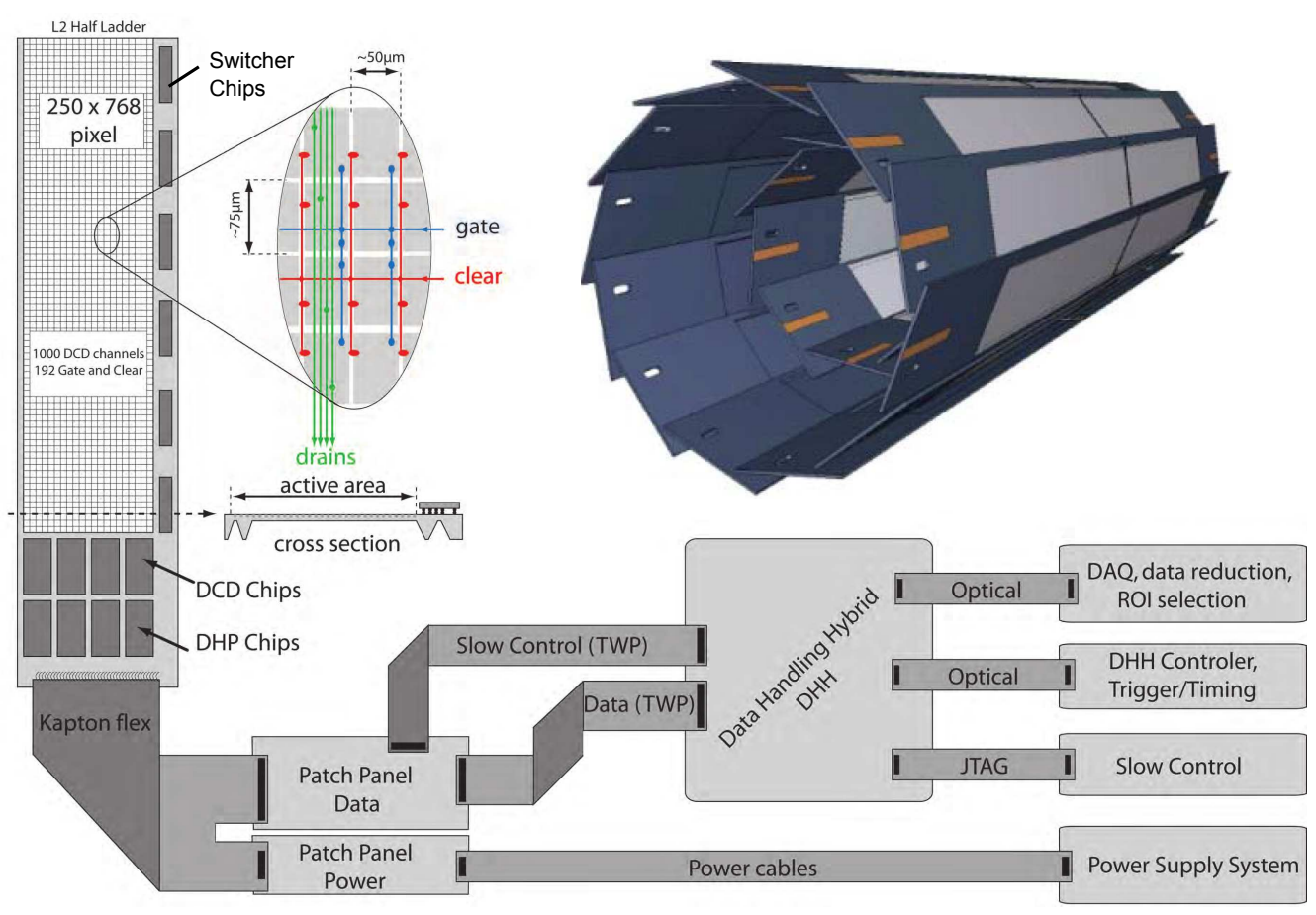

Figure 3: Overview over the individual components of the two-layer PXD (see insert). The schematics also shows the interconnections to the data acquisition system. 
A crucial step in the fabrication of the DEPFET sensors for Belle II is the back-thinning of the so-called "handle" wafer, which is bonded onto the "top" wafer, where the DEPFET structures are implemented. Before the bonding, the top wafer receives an electrically active backside implant. After direct wafer bonding the top wafer is thinned and polished to the desired thickness ( $75 \mu \mathrm{m}$ in the Belle II case). After processing of the top wafer, the bulk of the handle wafer is removed by deep anisotropic wet etching, which stops at the silicon oxide interface between the two wafers. Further details of the DEPFET processing, the electronics and interconnection technologies, together with discussions on the mechanics and cooling as well as the data acquisition system foreseen for the PXD can be found elsewhere $[6,8]$.

Recently, the first thinned $(50 \mu \mathrm{m})$ test matrices have become available. Apart from some yield problems in the production process (which re now identified and can be avoided in the future), the matrices behave as expected. They were tested with $\beta$ sources and also in test beams at CERN (120 GeV pions). The measured signal to noise ratio was about 40 at full readout speed for some smaller size matrices. A full prototype production is planned with the final geometrical layout of the DEPFET cells (optimized for radiation hardness), and the detailed evaluation of the prototypes (including the final electronics) is expected by the summer of 2013. The main production of the sensors for the PXD will be completed by early 2015, and the installation of the PXD in the Belle II interaction region is scheduled for the late fall of 2015.

\section{Conclusions}

Flavor physics continues to be the key to the puzzle of the observed matter-antimatter asymmetry in the universe, intimately connected to $\mathrm{CP}$ violation. The SuperKEKB project, together with a substantially upgraded detector, Belle II, with a physics program complementary to the LHC, will contribute to this fascinating chapter of particle physics, taking first data in late 2015, For Belle II a novel Si pixel vertex detector is being designed and constructed, based on the DEPFET technology, which will improve the vertex resolution obtained in Belle by about a factor of two. With the prospect of accumulating 50 times the presently available data by the year 2021, the Belle II Collaboration is looking forward for exciting discoveries of physics beyond the Standard Model.

\section{References}

[1] see, e.g., A. J. Buras, Flavour Physics and CP Violation, TUM-HEP-590/05.

[2] see, e.g., T. E. Browder, in "Hints for New Physics in Flavor Decays", KEK, March 20-21, 2009.

[3] S. Hashimoto et al., LoI for KEK Super B Factory, Part I: Machine, KEK-Report 2004-4, 2004.

[4] M. Bona et al., SuperB, a High-Luminosity Heavy Flavor Factory, INFN/AE-07/2, 2007.

[5] T. E. Browder et al., hep-ph/0802.3201.

[6] Belle II Collaboration, T. Abe e al., KEK-Report 2010-1 (2010).

[7] J. Kemmer, G. Lutz et al., NIM A253 (1987) 356; NIM A288 (1990) 92.

[8] see the webpage of the DEPFET Collaboration: http://www.depfet.org 